\title{
General decay of solutions for Kirchhoff type containing Balakrishnan-Taylor damping with a delay and acoustic boundary conditions
}

\author{
Mi Jin Lee', Daewook Kim² ${ }^{2 *}$ and Jong Yeoul Park'
}

\section{"Correspondence:}

kdw@seowon.ac.kr

${ }^{2}$ Department of Mathematics

Education, Seowon University,

Cheongju, 28674, South Korea

Full list of author information is

available at the end of the article

\begin{abstract}
In this paper, we are concerned with the general decay result of the quasi-linear wave equation for Kirchhoff type containing Balakrishnan-Taylor damping with a delay in the boundary feedback and acoustic boundary conditions.
\end{abstract}

MSC: 35L70; 35B40; 93D15

Keywords: general decay; Kirchhoff type; Balakrishnan-damping; boundary feedback; acoustic boundary condition

\section{Introduction}

Let $\Omega$ be a bounded domain of $R^{n}, n \geq 1$, with a smooth boundary $\Gamma=\Gamma_{0} \cup \Gamma_{1}$. Here $\Gamma_{0}$ and $\Gamma_{1}$ are closed and disjoint and $v=\left(v_{1}, v_{2}, \ldots, v_{n}\right)$ represents the unit outward normal to $\Gamma$. In this paper, we are concerned with the general decay of solutions of the quasilinear wave equation for Kirchhoff type containing Balakrishnan-Taylor damping with a delay and acoustic boundary condition,

$$
\begin{aligned}
& \left|u_{t}\right|^{\rho} u_{t t}-\left(a+b\|\nabla u\|^{2}+\sigma\left(\nabla u, \nabla u_{t}\right)\right) \Delta u-\Delta u_{t t} \\
& +\int_{0}^{t} g(t-s) \Delta u(s) d s+\left|u_{t}\right|^{q} u_{t}=|u|^{p} u \quad \text { in } \Omega \times(0,+\infty), \\
& u=0 \quad \text { on } \Gamma_{0} \times(0, \infty), \\
& \left(a+b\|\nabla u\|^{2}+\sigma\left(\nabla u, \nabla u_{t}\right)\right) \frac{\partial u}{\partial v}+\frac{\partial u_{t t}}{\partial v} \\
& -\int_{0}^{t} g(t-s) \frac{\partial u(s)}{\partial v} d s+\mu_{0} u_{t}(x, t)+\mu_{1} u_{t}(x, t-\tau(t))=h(x) y_{t} \\
& \text { on } \Gamma_{1} \times(0, \infty) \text {, } \\
& u_{t}+f(x) y_{t}+m(x) y=0 \quad \text { on } \Gamma_{1} \times(0, \infty), \\
& u(x, 0)=u_{0}(x), \quad u_{t}(x, 0)=u_{1}(x) \quad \text { in } \Omega, \\
& y(x, 0)=y_{0}(x) \text { in } \Gamma_{1},
\end{aligned}
$$

(c) 2016 Lee et al. This article is distributed under the terms of the Creative Commons Attribution 4.0 International License (http://creativecommons.org/licenses/by/4.0/), which permits unrestricted use, distribution, and reproduction in any medium, provided you give appropriate credit to the original author(s) and the source, provide a link to the Creative Commons license, and indicate if changes were made. 


$$
u_{t}(x, t-\tau(t))=f_{0}(x, t) \quad \text { in } \Gamma_{1},-\tau(0) \leq t \leq 0,
$$

where $\Delta=\sum_{j=1}^{N} \frac{\partial^{2}}{\partial x_{j}^{2}}$ and $\Omega \subset R^{n}, n \geq 1$ is a bounded domain with smooth boundary $\partial \Omega$ so that the divergence theorem can be applied. Here $a, b, \sigma, \rho, p, q>0$, the functions $f, m, h$ : $\Gamma_{1} \rightarrow R$ are essential bounded, $g$ represents the kernel of the memory term, $\mu_{0}, \mu_{1}$ are real numbers with $\mu_{0}>0, \mu_{1} \neq 0, \tau(t)>0$ represents the time-varying delay and initial datum $\left(u_{0}, u_{1}, f_{0}, y_{0}\right)$ belongs to a suitable space.

System (1.1)-(1.7) represents a nonlinear viscoelastic equation for Kirchhoff type containing Balakrishnan-Taylor damping with a time-varying delay and acoustic boundary conditions. The physical applications of the above system is related to the problem of noise control and suppression in practical applications. The noise sound propagates through some acoustic medium, for example, through air, in a room which is characterized by a bounded domain $\Omega$ whose walls, ceiling, and floor are described by the boundary conditions. This is the description of Wu in [1]. For a more physical explanation of the viscoelastic wave equations with acoustic boundary conditions, we refer the reader to [2-5]. The acoustic boundary conditions were introduced by Beale and Rosencrans in [6,7], where the authors proved the global existence and regularity of the linear problem. Time delays so often arise in many physical, chemical, biological, thermal, and economical phenomena because this phenomena depend not only on the present state but also on the past history of the system in a more complicated way. In recent years, differential equations with time delay effects have become an active area of research; see for example [8] and the references therein. To stabilize a hyperbolic system involving input delay terms, additional control terms will be necessary; see [9-11]. For instance in [9], the authors proved the boundary stabilization of a nonlinear viscoelastic equation with interior time-varying delay and nonlinear dissipative boundary feedback. For the model at hand, with Balakrishnan-Taylor damping $(\sigma \neq 0)$ and $g=0$, equation (1.1) is used to study the flutter panel equation and to the spillover problem, which was initially proposed by Balakrishnan and Taylor in 1989 [12], and Bass and Zes [13]. The related problems also were of concerned to You [14], Clark [15], Tatar and Zarai [16, 17], Mu et al. [18] and Lee et al. [19]. In particular, Wu [20] considered the following with Balakrishnan-Taylor damping and boundary conditions:

$$
\begin{aligned}
& u_{t t}-\left(a+b\|\nabla u\|^{2}+\sigma\left(\nabla u, \nabla u_{t}\right)\right) \Delta u+\int_{0}^{t} g(t-s) \Delta u(s) d s=|u|^{p-1} u \quad \text { in } \Omega \times(0,+\infty), \\
& u=0 \quad \text { on } \Gamma_{0} \times(0, \infty), \\
& \left(a+b\|\nabla u\|^{2}+\sigma\left(\nabla u, \nabla u_{t}\right)\right) \frac{\partial u}{\partial v}-\int_{0}^{t} g(t-s) \frac{\partial u(s)}{\partial v} d s+\alpha u_{t}=|u|^{k-1} u \quad \text { on } \Gamma_{1} \times(0, \infty), \\
& u(x, 0)=u_{0}(x), \quad u_{t}(x, 0)=u_{1}(x) \quad \text { in } \Omega .
\end{aligned}
$$

The author studied the general decay of solutions for a viscoelastic equation with Balakrishnan-Taylor damping. Zhang et al. [21], studied the global existence and asymptotic behavior of a nonlinear viscoelastic equation with interior time-varying delay and nonlinear dissipative boundary feedback as follows:

$$
\left|u_{t}\right|^{\rho} u_{t t}-\Delta u+\int_{0}^{t} h(t-s) \Delta u(s) d s+a u_{t}(x, t-\tau(t))=0, \quad x \in \Omega, t>0
$$




$$
\begin{aligned}
& u=0 \quad \text { on } \Gamma_{0} \times(0, \infty), \\
& \frac{\partial u}{\partial v}+g\left(u_{t}(x, t)\right)=0 \quad \text { on } \Gamma_{1} \times[0, \infty), \\
& u(x, 0)=u_{0}(x), \quad u_{t}(x, 0)=u_{1}(x), \quad x \in \Omega, \\
& u_{t}(x, t-\tau(t))=f_{0}(x, t), \quad x \in \Omega,-\tau(0) \leq t \leq 0 .
\end{aligned}
$$

Recently, Boukhatem and Benyatton in [22] were concerned with the local, uniqueness, global solution, and the decay of energy solution of the following model:

$$
\begin{aligned}
& u_{t t}+L u-\int_{0}^{t} g(t-s) L u(s) d s=|u|^{p-2} u \quad \text { in } \Omega \times(0, \infty) \text {, } \\
& u=0 \quad \text { on } \Gamma_{0} \times(0, \infty) \text {, } \\
& \frac{\partial u}{\partial v_{L}}-\int_{0}^{t} g(t-s) \frac{\partial u(s)}{\partial v_{L}} d s+\mu_{1} k_{1}\left(u_{t}(x, t)\right) \\
& +\mu_{2} k_{2}\left(u_{t}(x, t-\tau(t))\right)=h(x) z_{t} \quad \text { on } \Gamma_{1} \times(0, \infty), \\
& u_{t}+f(x) z_{t}+m(x) z=0 \quad \text { on } \Gamma_{1} \times(0, \infty) \text {, } \\
& u(x, 0)=u_{0}(x), \quad u_{t}(x, 0)=u_{1}(x) \quad \text { in } \Omega, \\
& z(x, 0)=z_{0}(x) \quad \text { on } \Gamma_{1} \text {. }
\end{aligned}
$$

Motivated by previous work, in this paper, we study the general decay of solutions for Kirchhoff type containing Balakrishnan-Taylor damping with a time-varying delay and acoustic boundary conditions. This is done by applying the idea presented in [23] with some necessary modification due to the nature of the problem treated here. To the best of our knowledge, there are no results for Kirchhoff type equations containing BalakrishnanTaylor damping with a delay and acoustic boundary conditions. Thus this work is meaningful. The plan of this paper is as following. In Section 2, we give some notation and material for our work. In Section 3, we prove the main result.

\section{Preliminaries}

In this section, we present some material that we shall use in order to present our result. Let $(\cdot, \cdot)$ be the scalar product in $L^{2}(\Omega)$, i.e.,

$$
(u, v)=\int_{\Omega} u(x) v(x) d x,
$$

and the corresponding norm $\|\cdot\|$, i.e., $\|u\|^{2}=(u, u)$. Also, we mean by $\|\cdot\|_{q}$ the $L^{q}(\Omega)$ norm for $1 \leq q \leq \infty$ and by $\|\cdot\|_{q, \Gamma_{1}}:\|\cdot\|_{\Gamma_{1}}$ the $L^{q}\left(\Gamma_{1}\right)$ norm. We denote by

$$
V=\left\{u \in H^{1}(\Omega) ; u=0 \text { on } \Gamma_{0}\right\}
$$

the closed subspace of $H^{1}(\Omega)$ equipped with the norm equivalent to the usual norm in $H^{1}(\Omega)$. The Poincaré inequality holds in $V$, i.e., $\forall u \in V$, there exists a constant $C_{*}$ such that

$$
\|u\|_{p} \leq C_{*}\|\nabla u\|, \quad 2 \leq p \leq \frac{2 N}{N-2}
$$


and there exists a constant $\tilde{C}_{*}>0$ such that

$$
\|u\|_{\Gamma_{1}} \leq \tilde{C}_{*}\|\nabla u\|, \quad \forall u \in V .
$$

For our study of problem (1.1)-(1.7) we will need the following assumptions. First, we assume that $\rho$ and $q$ satisfy

$$
0<\rho, q \leq \frac{2}{N-2} \quad \text { if } N \geq 3, \quad \rho, q>0 \quad \text { if } N=1,2,
$$

and $p$ satisfies

$$
0<p \leq \frac{4}{N-2} \quad \text { if } N \geq 3, \quad 2<p \quad \text { if } N=1,2 .
$$

With regard to the relation function $g(t)$, we assume that it verifies:

$$
\text { (H1) } g: R^{+} \rightarrow R^{+} \text {is a bounded } C^{1} \text { function satisfying }
$$

$$
g(0)>0, \quad a-\int_{0}^{\infty} g(s) d s:=l>0,
$$

and there exist a positive non-increasing $C^{1}$ function $\xi:[0, \infty) \rightarrow[0, \infty)$ and a positive constant $k$ such that

$$
\begin{aligned}
& g^{\prime}(t) \leq-\zeta(t) g(t), \quad\left|\frac{\zeta^{\prime}(t)}{\zeta(t)}\right| \leq k, \quad \zeta^{\prime}(t) \leq 0, \\
& \zeta(t)>0, \quad \int_{0}^{\infty} \zeta(s) d s=\infty, \quad \forall t \geq 0 .
\end{aligned}
$$

(H2) There exist three positive constants $f_{0 *}, m_{0}$, and $h_{0}$ such that

$$
f \geq f_{0 *}, \quad m \geq m_{0}, \quad \text { and } \quad h \geq h_{0} .
$$

(H3) For the time-varying delay, we assume as in [9] that there exist positive constants $\tau_{0}, \bar{\tau}$ such that

$$
0<\tau_{0} \leq \tau(t) \leq \bar{\tau}, \quad \forall t>0
$$

Furthermore, we assume that the delay satisfies

$$
\tau^{\prime}(t) \leq d<1, \quad \forall t>0,
$$

that

$$
\tau \in W^{2, \infty}([0, T]), \quad \forall T>0,
$$

and that $\mu_{0}, \mu_{1}$ satisfy

$$
\left|\mu_{1}\right|<\sqrt{1-d} \mu_{0}
$$


As in [9], let us introduce the function

$$
z(x, \rho, t)=u_{t}(x, t-\tau(t) \rho), \quad x \in \Omega, \rho \in(0,1), t>0
$$

Then problem (1.1)-(1.7) is equivalent to

$$
\begin{aligned}
& \left|u_{t}\right|^{\rho} u_{t t}-\left(a+b\|\nabla u\|^{2}+\sigma\left(\nabla u, \nabla u_{t}\right)\right) \Delta u-\Delta u_{t t} \\
& +\int_{0}^{t} g(t-s) \Delta u(s) d s+\left|u_{t}\right|^{q} u_{t}=|u|^{p} u \quad \text { in } \Omega \times(0,+\infty), \\
& u=0 \quad \text { on } \Gamma_{0} \times(0, \infty) \text {, } \\
& \left(a+b\|\nabla u\|^{2}+\sigma\left(\nabla u, \nabla u_{t}\right)\right) \frac{\partial u}{\partial v}+\frac{\partial u_{t t}}{\partial v} \\
& -\int_{0}^{t} g(t-s) \frac{\partial u(s)}{\partial v} d s+\mu_{0} u_{t}(x, t)+\mu_{1} z(x, 1, t)=h(x) y_{t} \quad \text { on } \Gamma_{1} \times(0, \infty), \\
& \tau(t) z_{t}(x, \rho, t)+\left(1-\tau^{\prime}(t) \rho\right) z_{\rho}(x, \rho, t)=0 \quad \text { on } \Gamma_{1} \times(0,1) \times(0, \infty), \\
& u_{t}+f(x) y_{t}+m(x) y=0 \quad \text { on } \Gamma_{1} \times(0, \infty) \text {, } \\
& u(x, 0)=u_{0}(x), \quad u_{t}(x, 0)=u_{1}(x) \quad \text { in } \Omega, \\
& y(x, 0)=y_{0}(x) \text { on } \Gamma_{1}, \\
& z(x, \rho, 0)=f_{0}(x,-\rho \tau(0)) \quad \text { on } \Gamma_{1} \times(0,1) .
\end{aligned}
$$

We now state the local existence result of problem (2.13)-(2.20), which can be established by combining with the argument of [6].

Theorem 2.1 Suppose that (2.3), (2.4), (H1)-(H3) hold and that $\left(u_{0}, u_{1}\right) \in H^{2}(\Omega) \cap V \times$ $V, y_{0} \in L^{2}\left(\Gamma_{1}\right)$, and $f_{0} \in L^{2}\left(\Gamma_{1} \times(0,1)\right)$.

Then, for any $T>0$, there exists a unique solution $(u, y, z)$ of problem (2.13)-(2.20) on $[0, T]$ such that

$$
\begin{array}{ll}
u \in L^{\infty}\left(0, T ; H^{2}(\Omega) \cap V\right), & u_{t} \in L^{\infty}(0, T ; V) \cap L^{q+2}(\Omega \times(0, T)) \cap L^{2}\left(\Gamma_{1} \times(0,1)\right), \\
h^{1 / 2} y \in L^{\infty}\left(0, T ; L^{2}\left(\Gamma_{1}\right)\right), & h^{1 / 2} y_{t} \in L^{2}\left(0, T ; L^{2}\left(\Gamma_{1}\right)\right) .
\end{array}
$$

\section{Main result}

In this section, we shall state and prove our main result. For this purpose, we define

$$
\begin{aligned}
J(t)= & \frac{1}{2}\left(a-\int_{0}^{t} g(s) d s\right)\|\nabla u(t)\|^{2}+\frac{b}{4}\|\nabla u(t)\|^{4}+\frac{1}{2}\left\|\nabla u_{t}(t)\right\|^{2}+\frac{1}{2}(g \circ \nabla u)(t) \\
& +\frac{\xi}{2} \int_{t-\tau(t)}^{t} \int_{\Gamma_{1}} e^{-\lambda(t-s)} u_{t}^{2}(s) d \Gamma d s+\frac{1}{2} \int_{\Gamma_{1}} m(x) h(x) y^{2}(t) d \Gamma \\
& -\frac{1}{p+2}\|u(t)\|_{p+2}^{p+2}
\end{aligned}
$$


and

$$
\begin{aligned}
I(t)= & \left(a-\int_{0}^{t} g(s) d s\right)\|\nabla u(t)\|^{2}+\frac{b}{2}\|\nabla u(t)\|^{4}+\left\|\nabla u_{t}(t)\right\|^{2}+(g \circ \nabla u)(t) \\
& +\xi \int_{t-\tau(t)}^{t} \int_{\Gamma_{1}} e^{-\lambda(t-s)} u_{t}^{2}(s) d \Gamma d s+\int_{\Gamma_{1}} m(x) h(x) y^{2}(t) d \Gamma-\|u(t)\|_{p+2}^{p+2},
\end{aligned}
$$

where $(g \circ \nabla u)(t)=\int_{\Omega} \int_{0}^{t} g(t-s)|u(t)-u(s)|^{2} d s d x$. We denote the modified energy functional $E(t)$ associated with problem (2.13)-(2.20) by

$$
\begin{aligned}
E(t)= & \frac{1}{\rho+2}\left\|u_{t}(t)\right\|_{\rho+2}^{\rho+2}+\frac{1}{2}\left(a-\int_{0}^{t} g(s) d s\right)\|\nabla u(t)\|^{2}+\frac{b}{4}\|\nabla u(t)\|^{4} \\
& +\frac{1}{2}\left\|\nabla u_{t}(t)\right\|^{2}+\frac{1}{2}(g \circ \nabla u)(t)+\frac{\xi}{2} \int_{t-\tau(t)}^{t} \int_{\Gamma_{1}} e^{-\lambda(t-s)} u_{t}^{2}(s) d \Gamma d s \\
& +\frac{1}{2} \int_{\Gamma_{1}} m(x) h(x) y^{2}(t) d \Gamma-\frac{1}{p+2}\|u(t)\|_{p+2}^{p+2} \\
= & \frac{1}{\rho+2}\left\|u_{t}(t)\right\|_{\rho+2}^{\rho+2}+J(t),
\end{aligned}
$$

where $\xi, \lambda$ are suitable positive constants.

Next, we will fix $\xi$ such that

$$
\begin{aligned}
& 2 \mu_{0}-\frac{\left|\mu_{1}\right|}{\sqrt{1-d}}-\xi>0, \quad \xi-\frac{\left|\mu_{1}\right|}{\sqrt{1-d}}>0, \\
& \lambda<\frac{1}{\bar{\tau}}\left|\log \frac{\left|\mu_{1}\right|}{\xi \sqrt{1-d}}\right| .
\end{aligned}
$$

Lemma 3.1 Let (2.8)-(2.11) be satisfied and $g$ satisfy (2.5). Then for the solution of problem (2.13)-(2.20), the energy functional defined by (3.3) satisfies

$$
\begin{aligned}
E^{\prime}(t) \leq & -\sigma\left(\frac{1}{2} \frac{d}{d t}\|\nabla u(t)\|^{2}\right)^{2}+\frac{1}{2}\left(g^{\prime} \circ \nabla u\right)(t)-\frac{1}{2} g(t)\|\nabla u(t)\|^{2} \\
& -\int_{\Omega}\left|u_{t}(t)\right|^{q+2} d x-C_{1} \int_{\Gamma_{1}}\left[u_{t}^{2}(x, t)+u_{t}^{2}(x, t-\tau(t))\right] d \Gamma \\
& -\frac{\lambda \xi}{2} \int_{t-\tau(t)}^{t} \int_{\Gamma_{1}} e^{-\lambda(t-s)} u_{t}^{2}(s) d \Gamma d s-\int_{\Gamma_{1}} h(x) f(x) y_{t}^{2}(t) d \Gamma,
\end{aligned}
$$

for some positive constant $C_{1}$.

Proof Differentiating (3.3) and using (2.14), we have

$$
\begin{aligned}
E^{\prime}(t)= & \int_{\Omega}\left|u_{t}(t)\right|^{\rho+1} u_{t t}(t) d x-\frac{1}{2} g(t)\|\nabla u(t)\|^{2} \\
& +\left(a-\int_{0}^{t} g(s) d s\right) \int_{\Omega} \nabla u(t) \nabla u_{t}(t) d x+\frac{b}{2}\|\nabla u(t)\|^{2} \int_{\Omega} \nabla u(t) \nabla u_{t}(t) d x \\
& +\int_{\Omega} \nabla u_{t}(t) \nabla u_{t t}(t) d x+\int_{0}^{t} g(t-s) \int_{\Omega} \nabla u_{t}(t)(\nabla u(t)-\nabla u(s)) d x d s
\end{aligned}
$$




$$
\begin{aligned}
& +\frac{1}{2} \int_{0}^{t} g^{\prime}(t-s) \int_{\Omega}|\nabla u(t)-\nabla u(s)|^{2} d x d s+\frac{\xi}{2} \int_{\Gamma_{1}} u_{t}^{2}(x, t) d \Gamma \\
& -\frac{\xi}{2} \int_{\Gamma_{1}} e^{-\lambda \tau(t)} u_{t}^{2}(x, t-\tau(t))\left(1-\tau^{\prime}(t)\right) d \Gamma-\frac{\lambda \xi}{2} \int_{t-\tau(t)}^{t} \int_{\Gamma_{1}} e^{-\lambda(t-s)} u_{t}^{2}(s) d \Gamma d s \\
& +\int_{\Gamma_{1}} h(x) m(x) y(t) y_{t}(t) d \Gamma-\int_{\Omega}|u(t)|^{p+1} u_{t}(t) d x \\
= & \int_{\Omega} u_{t}(t)\left[\left(a+b\|\nabla u(t)\|^{2}+\sigma\left(\nabla u(t), \nabla u_{t}(t)\right)\right) \Delta u(t)+\Delta u_{t t}(t)\right. \\
& \left.-\int_{0}^{t} g(t-s) \Delta u(s) d s-\int_{\Omega}\left|u_{t}(t)\right|^{q} u_{t}(t) d x+\int_{\Omega}|u(t)|^{p} u(t)\right] d x \\
& -\frac{1}{2} g(t)\|\nabla u(t)\|^{2}+\left(a-\int_{0}^{t} g(s) d s\right) \int_{\Omega} \nabla u(t) \nabla u_{t}(t) d x \\
& +\frac{b}{2}\|\nabla u(t)\|^{2} \int_{\Omega} \nabla u(t) \nabla u_{t}(t) d x+\int_{\Omega} \nabla u_{t}(t) \nabla u_{t t}(t) d x \\
& +\int_{0}^{t} g(t-s) \int_{\Omega} \nabla u_{t}(t)(\nabla u(t)-\nabla u(s)) d x d s \\
& +\frac{1}{2} \int_{0}^{t} g^{\prime}(t-s) \int_{\Omega}|\nabla u(t)-\nabla u(s)|^{2} d x d s+\frac{\xi}{2} \int_{\Gamma_{1}} u_{t}^{2}(t) d \Gamma \\
& +\frac{\xi}{2} \int_{\Gamma_{1}} e^{-\lambda \tau(t)} u_{t}^{2}(x, t-\tau(t))\left(1-\tau^{\prime}(t)\right) d \Gamma-\frac{\lambda \xi}{2} \int_{t-\tau(t)}^{t} \int_{\Gamma_{1}} e^{-\lambda(t-s)} u_{t}^{2}(s) d \Gamma d s \\
& +\int_{\Gamma_{1}} h(x) m(x) y(t) y_{t}(t) d \Gamma-\int_{\Omega}|u(t)|^{p+1} u_{t}(t) d x \\
= & \int_{\Gamma_{1}} h(x) y_{t}(t) u_{t}(t) d \Gamma-\mu_{0} \int_{\Gamma_{1}} u_{t}(x, t) u_{t}(t) d \Gamma-\mu_{1} \int_{\Gamma_{1}} u_{t}(x, t-\tau(t)) u_{t}(t) d \Gamma \\
& -\sigma\left(\frac{1}{2} \frac{d}{d t}\|\nabla u(t)\|^{2}\right)^{2}-\int_{\Omega}\left|u_{t}(t)\right|^{q+2} d x-\frac{1}{2} g(t) \int_{\Omega}|\nabla u(t)|^{2} d x \\
& +\frac{1}{2}(t-s) \int_{\Omega}|\nabla u(t)-\nabla u(s)|^{2} d x d s+\frac{\xi}{2} \int_{\Gamma_{1}} u_{t}^{2}(x, t) d \Gamma \\
& \int^{-\lambda \tau(t)} u_{t}^{2}(x, t-\tau(t))\left(1-\tau^{\prime}(t)\right) d \Gamma-\frac{\lambda \xi}{2} \int_{t-\tau(t)}^{t} \int_{\Gamma_{1}} e^{-\lambda(t-s)} u_{t}^{2}(s) d \Gamma d s \\
& \\
& \\
& \\
& \\
&
\end{aligned}
$$

Applying Young's inequality, we obtain

$$
\begin{aligned}
& -\mu_{1} \int_{\Gamma_{1}} u_{t}(x, t) u_{t}(x, t-\tau(t)) d \Gamma \\
& \quad \leq \frac{\left|\mu_{1}\right|}{2 \sqrt{1-d}} \int_{\Gamma_{1}} u_{t}^{2}(x, t) d \Gamma+\frac{\left|\mu_{1}\right| \sqrt{1-d}}{2} \int_{\Gamma_{1}} u_{t}^{2}(x, t-\tau(t)) d \Gamma,
\end{aligned}
$$

and using (2.7), we get

$$
\int_{\Gamma_{1}} h(x) y_{t}(t) u_{t}(t) d \Gamma+\int_{\Gamma_{1}} f(x) h(x) y_{t}^{2}(t) d \Gamma=-\int_{\Gamma_{1}} h(x) m(x) y_{t}(t) y(t) d \Gamma .
$$


Thus, from (3.6)-(3.8) and assumptions (2.8), (2.9), and (3.4), we arrive at

$$
\begin{aligned}
& E^{\prime}(t) \leq-\mu_{0} \int_{\Gamma_{1}} u_{t}^{2}(t) d \Gamma+\frac{\left|\mu_{1}\right|}{2 \sqrt{1-d}} \int_{\Gamma_{1}} u_{t}^{2}(t) d \Gamma+\frac{\left|\mu_{1}\right| \sqrt{1-d}}{2} \int_{\Gamma_{1}} u_{t}^{2}(x, t-\tau(t)) d \Gamma \\
& -\sigma\left(\frac{1}{2} \frac{d}{d t}\|\nabla u(t)\|^{2}\right)^{2}-\int_{\Omega}\left|u_{t}(t)\right|^{q+2} d x+\frac{1}{2}\left(g^{\prime} \circ \nabla u\right)(t)-\frac{1}{2} g(t)\|\nabla u(t)\|^{2} \\
& +\frac{\xi}{2} \int_{\Gamma_{1}} u_{t}^{2}(t) d \Gamma-\frac{\xi}{2} \int_{\Gamma_{1}} e^{-\lambda \tau(t)} u_{t}^{2}(x, t-\tau(t))\left(1-\tau^{\prime}(t)\right) d \Gamma \\
& -\frac{\lambda \xi}{2} \int_{t-\tau(t)}^{t} \int_{\Gamma_{1}} e^{-\lambda(t-s)} u_{t}^{2}(s) d \Gamma d s-\int_{\Gamma_{1}} f(x) h(x) y_{t}^{2}(t) d \Gamma \\
& \leq-\sigma\left(\frac{1}{2} \frac{d}{d t}\|\nabla u(t)\|^{2}\right)^{2}-\int_{\Omega}\left|u_{t}(t)\right|^{q+2} d x+\frac{1}{2}\left(g^{\prime} \circ \nabla u\right)(t)-\frac{1}{2} g(t)\|\nabla u(t)\|^{2} \\
& -\left(\mu_{0}-\frac{\left|\mu_{1}\right|}{2 \sqrt{1-d}}-\frac{\xi}{2}\right) \int_{\Gamma_{1}} u_{t}^{2}(t) d \Gamma \\
& -\left(\frac{\xi}{2}(1-d) e^{-\lambda \bar{\tau}}-\frac{\left|\mu_{1}\right| \sqrt{1-d}}{2}\right) \int_{\Gamma_{1}} u_{t}^{2}(x, t-\tau(t)) d \Gamma \\
& -\frac{\lambda \xi}{2} \int_{t-\tau(t)}^{t} \int_{\Gamma_{1}} e^{-\lambda(t-s)} u_{t}^{2}(s) d \Gamma d s-\int_{\Gamma_{1}} f(x) h(x) y_{t}^{2}(t) d \Gamma \\
& \leq-\sigma\left(\frac{1}{2} \frac{d}{d t}\|\nabla u(t)\|^{2}\right)^{2}-\int_{\Omega}\left|u_{t}(t)\right|^{q+2} d x+\frac{1}{2}\left(g^{\prime} \circ \nabla u\right)(t)-\frac{1}{2} g(t)\|\nabla u(t)\|^{2} \\
& -C_{1} \int_{\Gamma_{1}}\left[u_{t}(x, t)+u_{t}^{2}(x, t-\tau(t))\right] d \Gamma-\frac{\lambda \xi}{2} \int_{t-\tau(t)}^{t} \int_{\Gamma_{1}} e^{-\lambda(t-s)} u_{t}^{2}(s) d \Gamma d s \\
& -\int_{\Gamma_{1}} f(x) h(x) y_{t}^{2}(t) d \Gamma
\end{aligned}
$$

for some positive constant $C_{1}$.

Lemma 3.2 Let $\left(u_{0}, u_{1}\right) \in\left(H^{2}(\Omega) \cap V\right) \times V, y_{0} \in L^{2}\left(\Gamma_{1}\right), f_{0} \in L^{2}\left(\Gamma_{1} \times[-\tau(0), 0]\right)$, and $(u(t), y(t), z(t))$ be the solution of $(2.13)-(2.20)$. If $I(0)>0$ and

$$
\alpha=\frac{C_{*}^{p+2}}{l}\left(\frac{2(p+2)}{l p} E(0)\right)^{p / 2}<1
$$

then $I(t)>0$ for $t \in[0, T]$, where $I(t)$ is defined in (3.2).

Proof Since $I(0)>0$, there exists (by continuity of $u(t)$ ) $T^{*}<T$ such that

$$
I(t) \geq 0
$$

for all $t \in\left[0, T^{*}\right]$. Then (3.1), (3.2), and (3.10) give

$$
\begin{aligned}
J(t)= & \frac{p}{2(p+2)}\left[\left(a-\int_{0}^{t} g(s) d s\right)\|\nabla u(t)\|^{2}+\frac{b}{2}\|\nabla u(t)\|^{4}+\left\|\nabla u_{t}(t)\right\|^{2}+(g \circ \nabla u)(t)\right. \\
& \left.+\xi \int_{t-\tau(t)}^{t} \int_{\Gamma_{1}} e^{-\lambda(t-s)} u_{t}^{2}(s) d \Gamma d s+\int_{\Gamma_{1}} m(x) h(x) y^{2}(t) d \Gamma\right]+\frac{1}{p+2} I(t)
\end{aligned}
$$




$$
\begin{aligned}
\geq & \frac{p}{2(p+2)}\left[\left(a-\int_{0}^{t} g(s) d s\right)\|\nabla u(t)\|^{2}+\frac{b}{2}\|\nabla u(t)\|^{4}+\left\|\nabla u_{t}(t)\right\|^{2}+(g \circ \nabla u)(t)\right. \\
& \left.+\xi \int_{t-\tau(t)}^{t} \int_{\Gamma_{1}} e^{-\lambda(t-s)} u_{t}^{2}(s) d \Gamma d s+\int_{\Gamma_{1}} m(x) h(x) y^{2}(t) d \Gamma\right]
\end{aligned}
$$

Hence from (2.5), (3.3), (3.11), and Lemma 3.1, we can deduce that

$$
\begin{aligned}
l\|\nabla u(t)\|^{2} & \leq\left(a-\int_{0}^{t} g(s) d s\right)\|\nabla u(t)\|^{2} \leq \frac{2(p+2)}{p} J(t) \\
& \leq \frac{2(p+2)}{p} E(t) \leq \frac{2(p+2)}{p} E(0), \quad \forall t \in\left[0, T^{*}\right] .
\end{aligned}
$$

Exploiting (2.1), (3.9), and (3.12), we obtain

$$
\begin{aligned}
\|u(t)\|_{p+2}^{p+2} & \leq C_{*}^{p+2}\|\nabla u(t)\|^{p+2} \leq \frac{C_{*}^{p+2}}{l}\left(\frac{2(p+2)}{l p} E(0)\right)^{\frac{p}{2}} l\|\nabla u(t)\|^{2} \\
& \leq \alpha l\|\nabla u(t)\|^{2} \leq\left(a-\int_{0}^{t} g(s) d s\right)\|\nabla u(t)\|^{2}, \quad \forall t \in\left[0, T^{*}\right] .
\end{aligned}
$$

Consequently, we get

$$
\begin{aligned}
I(t)= & \left(a-\int_{0}^{t} g(s) d s\right)\|\nabla u(t)\|^{2}+\frac{b}{2}\|\nabla u(t)\|^{4}+\left\|\nabla u_{t}(t)\right\|^{2}+(g \circ \nabla u)(t) \\
& +\xi \int_{t-\tau(t)}^{t} \int_{\Gamma_{1}} e^{-\lambda(t-s)} u_{t}^{2}(s) d \Gamma d s+\int_{\Gamma_{1}} m(x) h(x) y^{2}(t) d \Gamma \\
& -\|u(t)\|_{p+2}^{p+2}>0, \quad \forall t \in\left[0, T^{*}\right] .
\end{aligned}
$$

Repeat this procedure and use the fact that

$$
\lim _{t \rightarrow T^{*}} \frac{C_{*}^{p+2}}{l}\left[\frac{2(p+2)}{l p} E(t)\right]^{\frac{p}{2}} \leq \alpha<1 .
$$

We can take $T^{*}=T$. Thus the proof is complete.

Theorem 3.1 Suppose that (2.1)-(2.4), (2.9)-(2.11), and (H1)-(H3) hold. If $\left(u_{0}, u_{1}\right) \in$ $\left(H^{2}(\Omega) \cap V\right) \times V, y_{0} \in L^{2}\left(\Gamma_{1}\right), f_{0} \in L^{2}\left(\Gamma_{1} \times[-\tau(0), 0]\right)$ and (3.9) is satisfied, then the solution $(u(t), y(t), z(t))$ of $(2.13)-(2.20)$ is bounded and global in time.

Proof It suffices to show that

$$
\|\nabla u(t)\|^{2}+\left\|\nabla u_{t}(t)\right\|^{2}+\xi \int_{t-\tau(t)}^{t} \int_{\Gamma_{1}} e^{-\lambda(t-s)} u_{t}^{2}(s) d \Gamma d s+\int_{\Gamma_{1}} m(x) h(x) y^{2}(t) d \Gamma
$$

is bounded independent of $t$. Under the hypotheses in Theorem 3.1, we see from Lemma 3.2 that $I(t)>0$ for all $t \geq 0$. Therefore 


$$
\begin{aligned}
J(t)= & \frac{p}{2(p+2)}\left[\left(a-\int_{0}^{t} g(s) d s\right)\|\nabla u(t)\|^{2}+\frac{b}{2}\|\nabla u(t)\|^{4}+\left\|\nabla u_{t}(t)\right\|^{2}+(g \circ \nabla u)(t)\right. \\
& \left.+\xi \int_{t-\tau(t)}^{t} \int_{\Gamma_{1}} e^{-\lambda(t-s)} u_{t}^{2}(s) d \Gamma d s+\int_{\Gamma_{1}} m(x) h(x) y^{2}(t) d \Gamma\right]+\frac{1}{p+2} I(t) \\
\geq & \frac{p}{2(p+2)}\left[\left(a-\int_{0}^{t} g(s) d s\right)\|\nabla u(t)\|^{2}+\frac{b}{2}\|\nabla u(t)\|^{4}+\left\|\nabla u_{t}(t)\right\|^{2}\right. \\
& \left.+(g \circ \nabla u)(t)+\xi \int_{t-\tau(t)}^{t} \int_{\Gamma_{1}} e^{-\lambda(t-s)} u_{t}^{2}(s) d \Gamma d s+\int_{\Gamma_{1}} m(x) h(x) y^{2}(t) d \Gamma\right] .
\end{aligned}
$$

Hence by $(\mathrm{H} 1)$ and the fact that $(g \circ \nabla u)(t)>0$, we can deduce that

$$
\begin{aligned}
& l\|\nabla u(t)\|^{2}+\frac{b}{2}\|\nabla u(t)\|^{4}+\left\|\nabla u_{t}(t)\right\|^{2} \\
& \quad+\xi \int_{t-\tau(t)}^{t} \int_{\Gamma_{1}} e^{-\lambda(t-s)} u_{t}^{2}(s) d \Gamma d s+\int_{\Gamma_{1}} m(x) h(x) y^{2}(t) d \Gamma \\
& \leq \quad\left(a-\int_{0}^{t} g(s) d s\right)\|\nabla u(t)\|^{2}+\frac{b}{2}\|\nabla u(t)\|^{4}+\left\|\nabla u_{t}(t)\right\|^{2} \\
& \quad+\xi \int_{t-\tau(t)}^{t} \int_{\Gamma_{1}} e^{-\lambda(t-s)} u_{t}^{2}(s) d \Gamma d s+\int_{\Gamma_{1}} m(x) h(x) y^{2}(t) d \Gamma \\
& \leq \frac{2(p+2)}{p} J(t), \quad \forall t \in[0, T] .
\end{aligned}
$$

Using Lemma 3.1 and (3.14), it follows that

$$
\begin{aligned}
& \frac{1}{\rho+2}\left\|u_{t}(t)\right\|_{\rho+2}^{\rho+2}+\frac{p}{2(p+2)}\left(l\|\nabla u(t)\|^{2}+\frac{b}{2}\|\nabla u(t)\|^{4}+\left\|\nabla u_{t}(t)\right\|^{2}\right. \\
& \left.\quad+\xi \int_{t-\tau(t)}^{t} \int_{\Gamma_{1}} e^{-\lambda(t-s)} u_{t}^{2}(s) d \Gamma d s+\int_{\Gamma_{1}} m(x) h(x) y^{2}(t) d \Gamma\right) \\
& \leq \frac{1}{\rho+2}\left\|u_{t}(t)\right\|_{\rho+2}^{\rho+2}+J(t)=E(t) \leq E(0) .
\end{aligned}
$$

Thus, there exists a constant $C>0$ depending $p$ and $l$ such that

$$
\begin{aligned}
& \|\nabla u(t)\|^{2}+\|\nabla u(t)\|^{4}+\left\|\nabla u_{t}(t)\right\|^{2} \\
& \quad+\xi \int_{t-\tau(t)}^{t} \int_{\Gamma_{1}} e^{-\lambda(t-s)} u_{t}^{2}(s) d \Gamma d s+\int_{\Gamma_{1}} m(x) h(x) y^{2}(t) d \Gamma \\
& \leq C E(t) \leq C E(0)<+\infty .
\end{aligned}
$$

This implies that the solution $(u(t), y(t), z(t))$ of (2.13)-(2.20) is bounded and global in time.

Now, we define

$$
L(t)=M E(t)+\varepsilon \Psi(t)+\Phi(t),
$$


where $M$ and $\varepsilon$ are positive constants which will be specified later and

$$
\begin{aligned}
\Psi(t)= & \frac{1}{\rho+1} \int_{\Omega}\left|u_{t}(t)\right|^{\rho} u_{t}(t) u(t) d x+\frac{\sigma}{4}\|\nabla u(t)\|^{4}+\int_{\Omega} \nabla u_{t}(t) \nabla u(t) d x \\
& +\int_{\Gamma_{1}} h(x) u(t) y(t) d \Gamma+\frac{1}{2} \int_{\Gamma_{1}} h(x) f(x) y^{2}(t) d \Gamma, \\
\Phi(t)= & -\frac{1}{\rho+1} \int_{\Omega}\left|u_{t}(t)\right|^{\rho} u_{t}(t) \int_{0}^{t} g(t-s)(u(t)-u(s)) d s d x \\
& -\int_{\Omega} \nabla u_{t}(t) \int_{0}^{t} g(t-s)(\nabla u(t)-\nabla u(s)) d s d x .
\end{aligned}
$$

Before we prove our main result, we need the following lemmas.

Lemma 3.3 Let $u \in L^{\infty}\left([0, T] ; H_{0}^{1}(\Omega)\right)$, then for any $\rho \geq 0$, we have

$$
\begin{aligned}
& \int_{\Omega}\left(\int_{0}^{t} g(t-s)(u(t)-u(s)) d s\right)^{\rho+2} d x \\
& \quad \leq(a-l)^{\rho+1} C_{*}^{\rho+2}\left(\frac{4(p+2) E(0)}{l p}\right)^{\rho / 2}(g \circ \nabla u)(t) .
\end{aligned}
$$

Proof By the Hölder inequality, (2.1), (2.5), and (3.12), we can deduce

$$
\begin{aligned}
& \int_{\Omega}\left(\int_{0}^{t} g(t-s)(u(t)-u(s)) d s\right)^{\rho+2} d x \\
& \quad \leq \int_{\Omega}\left(\int_{0}^{t} g(t-s) d s\right)^{\rho+1}\left(\int_{0}^{t} g(t-s)|u(t)-u(s)|^{\rho+2} d s\right) d x \\
& \quad \leq(a-l)^{\rho+1} C_{*}^{\rho+2} \int_{0}^{t} g(t-s)\|\nabla u(t)-\nabla u(s)\|^{\rho+2} d s \\
& \quad \leq(a-l)^{\rho+1} C_{*}^{\rho+2}\left(\frac{4(p+2) E(0)}{l p}\right)^{\rho / 2}(g \circ \nabla u)(t) .
\end{aligned}
$$

Lemma 3.4 Let $(u(t), y(t), z(t))$ be a solution of (2.13)-(2.20), then there exist two constants $\beta_{1}$ and $\beta_{2}$ such that

$$
\beta_{1} E(t) \leq L(t) \leq \beta_{2} E(t) .
$$

Proof By using (2.1), (3.12), and Young's inequality, we have

$$
\begin{aligned}
& \left.\left|\frac{1}{\rho+1} \int_{\Omega}\right| u_{t}(t)\right|^{\rho} u_{t}(t) u(t) d x \mid \\
& \quad \leq \frac{1}{\rho+2}\left\|u_{t}(t)\right\|_{\rho+2}^{\rho+2}+\frac{1}{(\rho+2)(\rho+1)}\|u(t)\|_{\rho+2}^{\rho+2} \\
& \quad \leq \frac{1}{\rho+2}\left\|u_{t}(t)\right\|_{\rho+2}^{\rho+2}+\frac{C_{*}^{\rho+2}}{(\rho+2)(\rho+1)}\|\nabla u(t)\|^{\rho+2} \\
& \quad \leq \frac{1}{\rho+2}\left\|u_{t}(t)\right\|_{\rho+2}^{\rho+2}+\frac{C_{*}^{\rho+2}}{(\rho+2)(\rho+1)}\left(\frac{2(p+2) E(0)}{l p}\right)^{\frac{\rho}{2}}\|\nabla u(t)\|^{2} \\
& \quad \leq \frac{1}{\rho+2}\left\|u_{t}(t)\right\|_{\rho+2}^{\rho+2}+\frac{\alpha_{1}}{(\rho+2)(\rho+1)}\|\nabla u(t)\|^{2},
\end{aligned}
$$


where $\alpha_{1}=C_{*}^{\rho+2}\left(\frac{2(p+2) E(0)}{l p}\right)^{\frac{\rho}{2}}$,

$$
\left|\int_{\Omega} \nabla u_{t}(t) \nabla u(t) d x\right| \leq \frac{1}{2}\left(\left\|\nabla u_{t}(t)\right\|^{2}+\|\nabla u(t)\|^{2}\right),
$$

and by (2.2) and (2.7), we can deduce

$$
\begin{aligned}
\left|\int_{\Gamma_{1}} h(x) u(t) y(t) d \Gamma\right| & =\int_{\Gamma_{1}} \frac{m(x) h(x) u(t)}{m(x)} y(t) d \Gamma \\
& \leq \frac{\|h\|_{\infty}^{1 / 2}\|m\|_{\infty}^{1 / 2}}{m_{0}}\left(\int_{\Gamma_{1}} h(x) m(x) y^{2}(x) d \Gamma\right)^{1 / 2}\left(\int_{\Gamma_{1}}|u(t)|^{2} d \Gamma\right)^{1 / 2} \\
& \leq \frac{\|h\|_{\infty}\|m\|_{\infty}}{2 m_{0}^{2}} \int_{\Gamma_{1}} h(x) m(x) y^{2}(x) d \Gamma+\frac{\tilde{C}_{*}}{2}\|\nabla u(t)\|^{2}
\end{aligned}
$$

Similarly, we obtain

$$
\begin{aligned}
& \left.\left|-\frac{1}{\rho+1} \int_{\Omega}\right| u_{t}(t)\right|^{\rho} u_{t}(t) \int_{0}^{t} g(t-s)(u(t)-u(s)) d s d x \mid \\
& \quad \leq \frac{1}{\rho+2}\left\|u_{t}(t)\right\|_{\rho+2}^{\rho+2}+\frac{C_{*}^{\rho+2}}{(\rho+2)(\rho+1)}(a-l)^{\rho+1} \int_{0}^{t} g(t-s)\|\nabla u(t)-\nabla u(s)\|^{\rho+2} d s \\
& \quad \leq \frac{1}{\rho+2}\left\|u_{t}(t)\right\|_{\rho+2}^{\rho+2}+\frac{(a-l)^{\rho+1} 2^{\frac{\rho}{2}} \alpha_{1}}{(\rho+1)(\rho+2)}(g \circ \nabla u)(t)
\end{aligned}
$$

and

$$
\begin{aligned}
& \left|-\int_{\Omega} \nabla u_{t}(t) \int_{0}^{t} g(t-s)(\nabla u(t)-\nabla u(s)) d s d x\right| \\
& \quad \leq \frac{1}{2}\left\|\nabla u_{t}(t)\right\|^{2}+\frac{a-l}{2}(g \circ \nabla u)(t) .
\end{aligned}
$$

Combining (3.15)-(3.17) and (3.20)-(3.24), we arrive at

$$
\begin{aligned}
\mid L(t) & -M E(t) \mid \\
\leq & \varepsilon|\Psi(t)|+|\Phi(t)| \\
\leq & \frac{\varepsilon}{\rho+2}\left\|u_{t}(t)\right\|_{\rho+2}^{\rho+2}+\frac{\varepsilon \alpha_{1}}{(\rho+2)(\rho+1)}\|\nabla u(t)\|^{2}+\frac{\varepsilon \sigma}{4}\|\nabla u(t)\|^{4}+\frac{\varepsilon}{2}\left\|\nabla u_{t}(t)\right\|^{2} \\
& +\frac{\varepsilon}{2}\|\nabla u(t)\|^{2}+\left(\frac{\varepsilon}{2 m_{0}} \frac{\|h\|_{\infty}\|m\|_{\infty}}{m_{0}}+\|f\|_{\infty}\right) \int_{\Gamma_{1}} h(x) m(x) y^{2}(t) d \Gamma \\
& +\frac{\varepsilon \tilde{C}_{*}}{2}\|\nabla u(t)\|^{2}+\frac{1}{\rho+2}\left\|u_{t}(t)\right\|_{\rho+2}^{\rho+2}+\frac{(a-l)^{\rho+1} 2^{\frac{\rho}{2}} \alpha_{1}}{(\rho+1)(\rho+2)}(g \circ \nabla u)(t) \\
& +\frac{1}{2}\left\|\nabla u_{t}(t)\right\|^{2}+\frac{a-l}{2}(g \circ \nabla u)(t) \\
= & \frac{\varepsilon+1}{\rho+2}\left\|u_{t}(t)\right\|_{\rho+2}^{\rho+2}+\varepsilon\left(\frac{\alpha_{1}}{(\rho+2)(\rho+1)}+\frac{1}{2}+\frac{\tilde{C}_{*}}{2}\right)\|\nabla u(t)\|^{2} \\
& +\frac{\varepsilon \sigma}{4 b} b\|\nabla u(t)\|^{4}+\frac{\varepsilon+1}{2}\left\|\nabla u_{t}(t)\right\|^{2}+\left(\frac{(a-l)^{\rho+1} 2^{\rho / 2} \alpha_{1}}{(\rho+1)(\rho+2)}+\frac{a-l}{2}\right)(g \circ \nabla u)(t)
\end{aligned}
$$




$$
\begin{aligned}
& +\left(\frac{\varepsilon}{2 m_{0}} \frac{\|h\|_{\infty}\|m\|_{\infty}}{m_{0}}+\|f\|_{\infty}\right) \int_{\Gamma_{1}} h(x) m(x) y^{2}(t) d \Gamma \\
\leq & C E(t),
\end{aligned}
$$

where $C$ is some positive constant. Choose $M>0$ sufficiently large and $\varepsilon$ small, there exist two positive constants $\beta_{1}$ and $\beta_{2}$ such that

$$
\beta_{1} E(t) \leq L(t) \leq \beta_{2} E(t)
$$

Thus the proof is complete.

Now, we state our main result.

Theorem 3.2 Suppose that (2.1)-(2.4), (2.9)-(2.11), and (H1)-(H3) hold. If $\left(u_{0}, u_{1}\right) \in$ $\left(H^{2}(\Omega) \cap V\right) \times V, y_{2} \in L^{2}\left(\Gamma_{1}\right), f_{0} \in\left(L^{2}\left(\Gamma_{1}\right) \times[-\tau(0), 0]\right)$ and (3.9) is satisfied. Then for each $t>0$, there exist positive constants $K$ and $v$ such that the energy of the solution for problem (2.13)-(2.20) satisfies

$$
E(t) \leq K e^{-v \int_{t_{0}}^{t} \zeta(s) d s}, \quad \forall t \geq t_{0} .
$$

Proof In order to obtain the energy result of $E(t)$, from Lemma 3.4, it suffices to prove that we have the estimate of $L(t)$. To this end, we need the derivative of $L(t)$. For this purpose, we estimate $\Psi^{\prime}(t)$. It follows from (3.16) and equations (2.13)-(2.17) that

$$
\begin{aligned}
\Psi^{\prime}(t)= & \int_{\Omega}\left|u_{t}(t)\right|^{\rho} u_{t t}(t) u(t) d x+\frac{1}{\rho+1} \int_{\Omega}\left|u_{t}(t)\right|^{\rho+2} d x \\
& +\sigma \int_{\Omega} \nabla u(t) \nabla u_{t}(t) \int_{\Omega} \nabla u(t) \nabla u(t) d x+\int_{\Omega} \nabla u_{t t}(t) \nabla u(t) d x+\int_{\Omega}\left|\nabla u_{t}(t)\right|^{2} d x \\
& +\int_{\Gamma_{1}} h(x) u_{t}(t) y(t) d \Gamma+\int_{\Gamma_{1}} h(x) u(t) y_{t}(t) d \Gamma+\int_{\Gamma_{1}} h(x) f(x) y(t) y_{t}(t) d \Gamma \\
= & \int_{\Omega}\left[\left|u_{t}(t)\right|^{\rho} u_{t t}(t)-\Delta u_{t t}(t)-\sigma\left(\nabla u_{t}(t), \nabla u(t)\right) \Delta u(t)\right] u(t) d x \\
& +\int_{\Gamma_{1}}\left(\frac{\partial u_{t t}(t)}{\partial v}+\sigma\left(\nabla u_{t}(t), \nabla u(t)\right) \frac{\partial u(t)}{\partial v}\right) u(t) d \Gamma \\
& +\frac{1}{\rho+1}\left\|u_{t}(t)\right\|_{\rho+2}^{\rho+2}+\int_{\Omega}\left|\nabla u_{t}(t)\right|^{2} d x+\int_{\Gamma_{1}} h(x) u_{t}(t) y(t) d \Gamma \\
& +\int_{\Gamma_{1}} h(x) u(t) y_{t}(t) d \Gamma+\int_{\Gamma_{1}} h(x) f(x) y(t) y_{t}(t) d \Gamma \\
& +\int_{\Gamma_{1}} h(x) f(x) y(t) y_{t}(t) d \Gamma \\
= & -\left(a+b\|\nabla u(t)\|^{2}\right) \int_{\Omega}|\nabla u(t)|^{2} d x+\int_{\Omega} \nabla u(t) \int_{0}^{t} g(t-s) \nabla u(s) d s d x \\
& -\int_{\Omega}\left|u_{t}(t)\right|^{q} u_{t}(t) u(t) d x+\int_{\Omega}|u(t)|^{p+2} d x \\
& +\int_{\Gamma_{1}}\left[\left(a+b\|\nabla u(t)\|^{2}+\sigma\left(\nabla u_{t}(t), \nabla u(t)\right)\right) \frac{\partial u(t)}{\partial v}\right.
\end{aligned}
$$




$$
\begin{aligned}
& \left.-\int_{0}^{t} g(t-s) \frac{\partial u(s)}{\partial v} d x+\frac{\partial u_{t t}(t)}{\partial v}\right] u(t) d \Gamma \\
& +\frac{1}{\rho+1}\left\|u_{t}(t)\right\|_{\rho+2}^{\rho+2}+\int_{\Omega}\left|\nabla u_{t}(t)\right|^{2} d x+\int_{\Gamma_{1}} h(x) u_{t}(t) y(t) d \Gamma \\
& +\int_{\Gamma_{1}} h(x) u(t) y_{t}(t) d \Gamma+\int_{\Gamma_{1}} h(x) f(x) y(t) y_{t}(t) d \Gamma \\
= & -\left(a+b\|\nabla u(t)\|^{2}\right) \int_{\Omega}|\nabla u(t)|^{2} d x+\int_{\Omega} \nabla u(t) \int_{0}^{t} g(t-s) \nabla u(s) d s d x \\
& -\int_{\Omega}\left|u_{t}(t)\right|^{q} u_{t}(t) u(t) d x+\int_{\Omega}|u(t)|^{p+2} d x-\mu_{0} \int_{\Gamma_{1}} u_{t}(x, t) u(t) d \Gamma \\
& -\mu_{1} \int_{\Gamma_{1}} u_{t}(x, t-\tau(t)) u(t) d \Gamma+\frac{1}{\rho+1}\left\|u_{t}(t)\right\|_{\rho+2}^{\rho+2}+\int_{\Omega}\left|\nabla u_{t}(t)\right|^{2} d x \\
& +2 \int_{\Gamma_{1}} h(x) u(t) y_{t}(t) d \Gamma-\int_{\Gamma_{1}} h(x) m(x) y^{2}(t) d \Gamma .
\end{aligned}
$$

Now, we estimate the right hand side of (3.26). By using (2.1), (2.2), (2.7), and Young's inequality, for any $\eta>0$, we obtain

$$
\begin{aligned}
& \left|\int_{\Omega} \nabla u(t) \int_{0}^{t} g(t-s) \nabla u(s) d s\right| \\
& \quad=\int_{\Omega} \int_{0}^{t} g(t-s)(\nabla u(s)-\nabla u(t)) \nabla u(t) d s d x+\int_{0}^{t} g(s) d s \int_{\Omega}|\nabla u(t)|^{2} d x \\
& \quad \leq(1+\eta) \int_{0}^{t} g(s) d s\|\nabla u(t)\|^{2}+\frac{1}{4 \eta}(g \circ \nabla u)(t) \\
& \quad \leq(1+\eta)(a-l)\|\nabla u(t)\|^{2}+\frac{1}{4 \eta}(g \circ \nabla u)(t), \\
& \left.\left|-\int_{\Omega}\right| u_{t}(t)\right|^{q} u_{t}(t) u(t) d x \mid \\
& \quad \leq \eta C_{*}^{2} \int_{\Omega}|\nabla u(t)|^{2} d x+\frac{1}{4 \eta}\left|u_{t}(t)\right|^{2(q+1)} d x \\
& \quad \leq \eta C_{*}^{2} \int_{\Omega}|\nabla u(t)|^{2} d x+\frac{\alpha_{2}}{4 \eta} \int_{\Omega}\left|\nabla u_{t}(t)\right|^{2} d x,
\end{aligned}
$$

where $\alpha_{2}=C_{*}^{2(q+1)}\left(\frac{2(p+2) E(0)}{p}\right)^{q}$,

$$
\begin{aligned}
& \left|-\mu_{0} \int_{\Gamma_{1}} u_{t}(x, t) u(t) d \Gamma\right| \\
& \quad \leq \eta \mu_{0} \tilde{C}_{*}^{2} \int_{\Omega}|\nabla u(t)|^{2} d x+\frac{\mu_{0}}{4 \eta} \int_{\Gamma_{1}} u_{t}^{2}(t) d \Gamma \\
& \left|-\mu_{1} \int_{\Gamma_{1}} u_{t}(x, t-\tau(t)) u(t) d \Gamma\right| \\
& \leq \eta\left|\mu_{1}\right| \tilde{C}_{*}^{2} \int_{\Omega}|\nabla u(t)|^{2} d x+\frac{\left|\mu_{1}\right|}{4 \eta} \int_{\Gamma_{1}} u_{t}^{2}(x, t-\tau(t)) d \Gamma
\end{aligned}
$$


and

$$
\begin{aligned}
\left|\int_{\Gamma_{1}} h(x) u(t) y_{t}(t) d \Gamma\right| & \leq\|h\|_{\infty}^{1 / 2}\left(\int_{\Gamma_{1}} h(x) y_{t}^{2}(t) d \Gamma\right)^{1 / 2}\left(\int_{\Gamma_{1}}|u(t)|^{2} d \Gamma\right)^{1 / 2} \\
& \leq \eta \tilde{C}_{*}^{2} \int_{\Omega}|\nabla u(t)|^{2} d x+\frac{\|h\|_{\infty}}{4 \eta f_{0 *}} \int_{\Gamma_{1}} h(x) f(x) y_{t}^{2}(t) d \Gamma .
\end{aligned}
$$

Substitution of (3.27)-(3.31) into (3.26) yields

$$
\begin{aligned}
\Psi^{\prime}(t) \leq & -\left(a+b\|\nabla u(t)\|^{2}\right) \int_{\Omega}|\nabla u(t)|^{2} d x+(1+\eta)(a-l) \int_{\Omega}|\nabla u(t)|^{2} d x \\
& +\frac{1}{4 \eta}(g \circ \nabla u)(t)+\eta C_{*}^{2} \int_{\Omega}|\nabla u(t)|^{2} d x+\frac{\alpha_{2}}{4 \eta} \int_{\Omega}\left|\nabla u_{t}(t)\right|^{2} d x \\
& +\int_{\Omega}|u(t)|^{p+2} d x+\eta \mu_{0} \tilde{C}_{*}^{2} \int_{\Omega}|\nabla u(t)|^{2} d x+\frac{\mu_{0}}{4 \eta} \int_{\Gamma_{1}} u_{t}^{2}(t) d \Gamma \\
& +\eta\left|\mu_{1}\right| \tilde{C}_{*}^{2} \int_{\Omega}|\nabla u(t)|^{2} d x+\frac{\left|\mu_{1}\right|}{4 \eta} \int_{\Gamma_{1}} u_{t}^{2}(x, t-\tau(t)) d \Gamma \\
& +\frac{1}{\rho+1}\left\|u_{t}(t)\right\|_{\rho+2}^{\rho+2}+\int_{\Omega}\left|\nabla u_{t}(t)\right|^{2} d x+\eta \tilde{C}_{*}^{2} \int_{\Omega}|\nabla u(t)|^{2} d x \\
& +\frac{\|h\|_{\infty}}{2 \eta f_{0 *}} \int_{\Gamma_{1}} h(x) f(x) y_{t}^{2}(t) d \Gamma-\int_{\Gamma_{1}} h(x) m(x) y^{2}(t) d \Gamma \\
= & \frac{1}{\rho+1}\left\|u_{t}(t)\right\|_{\rho+2}^{\rho+2}-\left[\left(a+b\|\nabla u(t)\|^{2}\right)-(1+\eta)(a-l)-\eta C_{*}^{2}-\eta \mu_{0} \tilde{C}_{*}^{2}\right. \\
& \left.-\eta\left|\mu_{1}\right| \tilde{C}_{*}^{2}-\eta \tilde{C}_{*}^{2}\right]\|\nabla u(t)\|^{2}+\left(\frac{\alpha_{2}}{4 \eta}+1\right)\left\|\nabla u_{t}(t)\right\|^{2}+\frac{1}{4 \eta}(g \circ \nabla u)(t) \\
& +\|u(t)\|_{p+2}^{p+2}+\frac{\mu_{0}}{4 \eta} \int_{\Gamma_{1}} u_{t}^{2}(t) d \Gamma+\frac{\left|\mu_{1}\right|}{4 \eta} \int_{\Gamma_{1}} u_{t}^{2}(x, t-\tau(t)) d \Gamma \\
& +\frac{\|h\|_{\infty}}{2 \eta f_{0 *}} \int_{\Gamma_{1}} h(x) f(x) y_{t}^{2}(t) d \Gamma-\int_{\Gamma_{1}} h(x) m(x) y^{2}(t) d \Gamma . \\
& \\
&
\end{aligned}
$$

Next, we would like to estimate $\Phi^{\prime}(t)$. Taking the derivative of $\Phi(t)$ in (3.17) and using (2.13)-(2.17), we can deduce that

$$
\begin{aligned}
\Phi^{\prime}(t)= & -\int_{\Omega}\left|u_{t}(t)\right|^{\rho} u_{t t}(t) \int_{0}^{t} g(t-s)(u(t)-u(s)) d s d x \\
& -\frac{1}{\rho+1} \int_{\Omega}\left|u_{t}(t)\right|^{\rho} u_{t}(t) \int_{0}^{t} g^{\prime}(t-s)(u(t)-u(s)) d s d x \\
& -\frac{1}{\rho+1} \int_{\Omega}\left|u_{t}(t)\right|^{\rho} u_{t}(t) \int_{0}^{t} g(t-s) u_{t}(t) d s d x \\
& -\int_{\Omega} \nabla u_{t t}(t) \int_{0}^{t} g(t-s)(\nabla u(t)-\nabla u(s)) d s d x \\
& -\int_{\Omega} \nabla u_{t}(t) \int_{0}^{t} g^{\prime}(t-s)(\nabla u(t)-\nabla u(s)) d s d x \\
& -\int_{\Omega} \nabla u_{t}(t) \int_{0}^{t} g(t-s) \nabla u_{t}(t) d s d x
\end{aligned}
$$




$$
\begin{aligned}
& =-\int_{\Omega}\left[\left(a+b\|\nabla u(t)\|^{2}\right) \Delta u(t)+\sigma\left(\nabla u(t), \nabla u_{t}(t)\right) \Delta u(t)-\int_{0}^{t} g(t-s) u(s) d s\right. \\
& \left.-\left|u_{t}(t)\right|^{q} u_{t}(t)+|u(t)|^{p} u(t)\right] \int_{0}^{t} g(t-s)(u(t)-u(s)) d s d x \\
& -\int_{\Gamma_{1}} \frac{\partial u_{t t}(t)}{\partial v} \int_{0}^{t} g(t-s)(u(t)-u(s)) d s d \Gamma \\
& -\int_{\Omega} \nabla u_{t}(t) \int_{0}^{t} g^{\prime}(t-s)(\nabla u(t)-\nabla u(s)) d s d x \\
& -\int_{\Omega} \nabla u_{t}(t) \int_{0}^{t} g(t-s) \nabla u_{t}(t) d s d x \\
& -\frac{1}{\rho+1} \int_{\Omega}\left|u_{t}(t)\right|^{\rho} u_{t}(t) \int_{0}^{t} g^{\prime}(t-s)(u(t)-u(s)) d s d x \\
& -\frac{1}{\rho+1} \int_{\Omega}\left|u_{t}(t)\right|^{\rho} u_{t}(t) \int_{0}^{t} g(t-s) u_{t}(t) d s d x \\
& =\left(a+b\|\nabla u(t)\|^{2}\right) \int_{\Omega} \nabla u(t) \int_{0}^{t} g(t-s)(\nabla u(t)-\nabla u(s)) d s d x \\
& +\sigma \int_{\Omega} \nabla u(t) \nabla u_{t}(t) \int_{\Omega} \nabla u(t) \int_{0}^{t} g(t-s)(\nabla u(t)-\nabla u(s)) d s d x \\
& -\int_{\Omega} \int_{0}^{t} g(t-s) \nabla u(s) d s \int_{0}^{t} g(t-s)(\nabla u(t)-\nabla u(s)) d s d x \\
& -\int_{\Gamma_{1}}\left(h(x) y_{t}(t)-\mu_{0} u_{t}(x, t)-\mu_{1} u_{t}(x, t-\tau(t))\right) \int_{0}^{t} g(t-s)(u(t)-u(s)) d s d \Gamma \\
& +\int_{\Omega}\left|u_{t}(t)\right|^{q} u_{t}(t) \int_{0}^{t} g(t-s)(u(t)-u(s)) d s d x \\
& -\int_{\Omega}|u(t)|^{p} u(t) \int_{0}^{t} g(t-s)(u(t)-u(s)) d s d x \\
& -\int_{\Omega} \nabla u_{t}(t) \int_{0}^{t} g^{\prime}(t-s)(\nabla u(t)-\nabla u(s)) d s d x \\
& -\int_{\Omega} \nabla u_{t}(t) \int_{0}^{t} g(t-s) \nabla u_{t}(t) d s d x \\
& -\frac{1}{\rho+1} \int_{\Omega}\left|u_{t}(t)\right|^{\rho} u_{t}(t) \int_{0}^{t} g^{\prime}(t-s)(u(t)-u(s)) d s d x \\
& -\frac{1}{\rho+1} \int_{\Omega}\left|u_{t}(t)\right|^{\rho} u_{t}(t) \int_{0}^{t} g(t-s) u_{t}(t) d s d x
\end{aligned}
$$

Now, we will estimate the right hand side of (3.33). From Lemma 3.3, (2.1), (2.2), (2.5), (3.5), (3.12), and Young's inequality, for any $\eta>0$, we have the following inequalities:

$$
\begin{aligned}
& \left|\int_{\Omega}\left(a+b\|\nabla u(t)\|^{2}\right) \nabla u(t) \int_{0}^{t} g(t-s)(\nabla u(t)-\nabla u(s)) d s d x\right| \\
& \quad \leq\left|\int_{\Omega}\left(a+b \frac{2(p+2)}{l p} E(0)\right) \nabla u(t) \int_{0}^{t} g(t-s)(\nabla u(t)-\nabla u(s)) d s d x\right| \\
& \quad \leq \eta\|\nabla u(t)\|^{2}+\frac{a-l}{4 \eta}\left(a+b \frac{2(p+2)}{l p} E(0)\right)^{2}(g \circ \nabla u)(t),
\end{aligned}
$$




$$
\begin{aligned}
& \left|\sigma \int_{\Omega} \nabla u(t) \nabla u_{t}(t) d x \int_{\Omega} \nabla u(t) \int_{0}^{t} g(t-s)(\nabla u(t)-\nabla u(s)) d s d x\right| \\
& \leq \sigma^{2}\left(\int_{\Omega} \nabla u(t) \nabla u_{t}(t) d x\right)^{2} \eta\|\nabla u(t)\|^{2} \\
& +\frac{1}{4 \eta} \int_{\Omega}\left(\int_{0}^{t} g(t-s)(\nabla u(t)-\nabla u(s)) d s\right)^{2} d x \\
& \leq \frac{2(p+2) \sigma^{2}}{p} E(0)\left(\int_{\Omega} \nabla u(t) \nabla u_{t}(t) d x\right)^{2}+\frac{a-l}{4 \eta}(g \circ \nabla u)(t) \\
& \leq-\frac{2(p+2) \sigma}{p} E(0) E^{\prime}(t)+\frac{a-l}{4 \eta}(g \circ \nabla u)(t), \\
& \left|-\int_{\Omega} \int_{0}^{t} g(t-s) \nabla u(s) d s \int_{0}^{t} g(t-s)(\nabla u(t)-\nabla u(s)) d s d x\right| \\
& \leq\left(2 \eta+\frac{1}{4 \eta}\right)(a-l)(g \circ \nabla u)(t)+2 \eta(a-l)^{2}\|\nabla u(t)\|^{2}, \\
& \left|-\int_{\Gamma_{1}} h(x) y_{t}(t) \int_{0}^{t} g(t-s)(u(t)-u(s)) d s d \Gamma\right| \\
& \leq \frac{\eta\|h\|_{\infty}}{f_{0 *}} \int_{\Gamma_{1}} h(x) f(x) y_{t}^{2}(t) d \Gamma+\frac{(a-l) \tilde{C}_{*}^{2}}{4 \eta}(g \circ \nabla u)(t), \\
& \left|-\int_{\Gamma_{1}} \mu_{0} u_{t}(x, t) \int_{0}^{t} g(t-s)(u(t)-u(s)) d s d \Gamma\right| \\
& \leq \eta \mu_{0} \int_{\Gamma_{1}}\left|u_{t}(x, t)\right|^{2} d \Gamma+\frac{\mu_{0}(a-l) \tilde{C}_{*}^{2}}{4 \eta}(g \circ \nabla u)(t), \\
& \left|-\int_{\Gamma_{1}} \mu_{1} u_{t}(x, t-\tau(t)) \int_{0}^{t} g(t-s)(u(t)-u(s)) d s d \Gamma\right| \\
& \leq \eta\left|\mu_{1}\right| \int_{\Gamma_{1}} u_{t}^{2}(x, t-\tau(t)) d \Gamma+\frac{\left|\mu_{1}\right|(a-l) \tilde{C}_{*}^{2}}{4 \eta}(g \circ \nabla u)(t), \\
& \left.\left|\int_{\Omega}\right| u_{t}(t)\right|^{q} u_{t}(t) \int_{0}^{t} g(t-s)(u(t)-u(s)) d s d x \mid \\
& \leq \eta \alpha_{2}\left\|\nabla u_{t}(t)\right\|^{2}+\frac{(a-l) \tilde{C}_{*}^{2}}{4 \eta}(g \circ \nabla u)(t),
\end{aligned}
$$

where $\alpha_{2}=C_{*}^{2(q+1)}\left(\frac{2(p+2) E(0)}{p}\right)^{q}$,

$$
\begin{gathered}
\left.\left|-\int_{\Omega}\right| u_{t}(t)\right|^{p} u(t) \int_{0}^{t} g(t-s)(u(t)-u(s)) d s d x \mid \\
\leq \eta \alpha_{3}\|\nabla u(t)\|^{2}+\frac{(a-l) \tilde{C}_{*}^{2}}{4 \eta}(g \circ \nabla u)(t),
\end{gathered}
$$

where $\alpha_{3}=C_{*}^{2(p+1)}\left(\frac{2(p+2) E(0)}{l p}\right)^{p}$

$$
\begin{aligned}
& \left|-\int_{\Omega} \nabla u_{t}(t) \int_{0}^{t} g^{\prime}(t-s)(\nabla u(t)-\nabla u(s)) d s d x\right| \\
& \quad \leq \eta\left\|\nabla u_{t}(t)\right\|^{2}-\frac{g(0)}{4 \eta}\left(g^{\prime} \circ \nabla u\right)(t),
\end{aligned}
$$




$$
\begin{aligned}
\mid- & \frac{1}{\rho+1} \int_{\Omega}\left|u_{t}(t)\right|^{\rho} u_{t}(t) \int_{0}^{t} g^{\prime}(t-s)(u(t)-u(s)) d s d x \mid \\
& \leq \frac{\eta}{\rho+1} C_{*}^{2(\rho+1)}\left(\frac{2(p+2) E(0)}{p}\right)^{\rho}\left\|\nabla u_{t}(t)\right\|^{2}-\frac{g(0) C_{*}^{2}}{4 \eta(\rho+1)}\left(g^{\prime} \circ \nabla u\right)(t) \\
& =\frac{\eta \alpha_{4}}{\rho+1}\left\|\nabla u_{t}(t)\right\|^{2}-\frac{g(0) C_{*}^{2}}{4 \eta(\rho+1)}\left(g^{\prime} \circ \nabla u\right)(t),
\end{aligned}
$$

where $\alpha_{4}=C_{*}^{2(\rho+1)}\left(\frac{2(p+2) E(0)}{p}\right)^{\rho}$. Thus from (3.33)-(3.43) we arrive at

$$
\begin{aligned}
& \Phi^{\prime}(t) \leq \eta\|\nabla u(t)\|^{2}+\frac{a-l}{4 \eta}\left(a+b \frac{2(p+2)}{l p} E(0)\right)^{2}(g \circ \nabla u)(t) \\
& -\frac{2(p+2) \sigma}{p} E(0) E^{\prime}(t)+\frac{a-l}{4 \eta}(g \circ \nabla u)(t) \\
& +\left(2 \eta+\frac{1}{4 \eta}\right)(a-l)(g \circ \nabla u)(t)+2 \eta(a-l)^{2}\|\nabla u(t)\|^{2} \\
& +\frac{\eta\|h\|_{\infty}}{f_{0}} \int_{\Gamma_{1}} h(x) f(x) y_{t}^{2}(t) d \Gamma+\frac{(a-l) \tilde{C}_{*}^{2}}{4 \eta}(g \circ \nabla u)(t) \\
& +\eta \mu_{0}\left\|u_{t}(x, t)\right\|^{2}+\frac{\mu_{0}(a-l) \tilde{C}_{*}^{2}}{4 \eta}(g \circ \nabla u)(t) \\
& +\eta\left|\mu_{1}\right| \int_{\Gamma_{1}} u_{t}^{2}(x, t-\tau(t)) d \Gamma+\frac{\left|\mu_{1}\right|(a-l) \tilde{C}_{*}^{2}}{4 \eta}(g \circ \nabla u)(t) \\
& +\eta \alpha_{2}\left\|\nabla u_{t}(t)\right\|^{2}+\frac{(a-l) \tilde{C}_{*}^{2}}{4 \eta}(g \circ \nabla u)(t)+\eta \alpha_{3}\|\nabla u(t)\|^{2}+\frac{(a-l) \tilde{C}_{*}^{2}}{4 \eta}(g \circ \nabla u)(t) \\
& +\eta\left\|\nabla u_{t}(t)\right\|^{2}-\frac{g(0)}{4 \eta}\left(g^{\prime} \circ \nabla u\right)(t)-\left(\int_{0}^{t} g(s) d s\right)\left\|\nabla u_{t}(t)\right\|^{2} \\
& +\frac{\eta \alpha_{4}}{\rho+1}\left\|\nabla u_{t}(t)\right\|^{2}-\frac{g(0) C_{*}^{2}}{4 \eta(\rho+1)}\left(g^{\prime} \circ \nabla u\right)(t)-\left(\int_{0}^{t} g(s) d s\right) \frac{1}{\rho+1}\left\|u_{t}(t)\right\|_{\rho+2}^{\rho+2} \\
& =-\left(\int_{0}^{t} g(s) d s\right) \frac{1}{\rho+1}\left\|u_{t}(t)\right\|_{\rho+2}^{\rho+2}+\eta\left(1+\alpha_{3}+2(a-l)^{2}\right)\|\nabla u(t)\|^{2} \\
& -\left[\int_{0}^{t} g(s) d s-\eta\left(\alpha_{2}+\frac{\alpha_{4}}{\rho+1}\right)\right]\left\|\nabla u_{t}(t)\right\|^{2} \\
& +\frac{(a-l)}{4 \eta}\left[\left(a+b \frac{2(p+2)}{l p} E(0)\right)^{2}+2+3 \tilde{C}_{*}^{2}+8 \eta^{2}+\mu_{0} \tilde{C}_{*}^{2}+\left|\mu_{1}\right| \tilde{C}_{*}^{2}\right] \\
& \times(g \circ \nabla u)(t)-\frac{g(0)}{4 \eta}\left(1+\frac{C_{*}^{2}}{\rho+1}\right)\left(g^{\prime} \circ \nabla u\right)(t)-\frac{2(p+2) \sigma}{p} E(0) E^{\prime}(t) \\
& +\frac{\eta\|h\|_{\infty}}{f_{0 *}} \int_{\Gamma_{1}} h(x) f(x) y_{t}^{2}(t) d \Gamma+\eta \mu_{0} \int_{\Gamma_{1}}\left|u_{t}(x, t)\right|^{2} d \Gamma \\
& +\eta\left|\mu_{1}\right| \int_{\Gamma_{1}} u_{t}^{2}(x, t-\tau(t)) d \Gamma \text {. }
\end{aligned}
$$

Since $g(t)$ is positive and continuous, for any $t_{0}>0$ we see that

$$
\int_{0}^{t} g(s) d s \geq \int_{0}^{t_{0}} g(s) d s \equiv g_{0}, \quad \forall t \geq t_{0} .
$$


Hence from (3.5), (3.15), (3.32), and (3.44), we conclude that for any $t \geq t_{0}>0$,

$$
\begin{aligned}
& L^{\prime}(t)=M E^{\prime}(t)+\varepsilon \Psi^{\prime}(t)+\Phi^{\prime}(t) \\
& \leq-\frac{1}{\rho+1}\left(g_{0}-\varepsilon\right)\left\|u_{t}(t)\right\|_{\rho+2}^{\rho+2} \\
& -\left[\frac{M g(0)}{2}-\varepsilon(1+\eta)(a-l)-\varepsilon \eta\left(C_{*}^{2}+\mu_{0} \tilde{C}_{*}^{2}+\tilde{C}_{*}^{2}+\left|\mu_{1}\right| \tilde{C}_{*}^{2}\right)\right. \\
& \left.-\eta\left(1+\alpha_{3}\right)-2 \eta(a-l)^{2}\right]\|\nabla u(t)\|^{2}-\varepsilon\left(a+b\|\nabla u(t)\|^{2}\right)\|\nabla u(t)\|^{2} \\
& -\left[g_{0}-\eta\left(\alpha_{2}+\frac{\alpha_{4}}{\rho+1}\right)-\varepsilon\left(\frac{\alpha_{2}}{4 \eta}+1\right)\right]\left\|\nabla u_{t}(t)\right\|^{2} \\
& +\frac{1}{4 \eta}\left[\varepsilon+(a-l)\left(a+b \frac{2(p+2)}{l p} E(0)\right)^{2}\right. \\
& +(a-l)\left(2+3 \tilde{C}_{*}^{2}+8 \eta^{2}+\mu_{0} \tilde{C}_{*}^{2}+\left|\mu_{1}\right| \tilde{C}_{*}^{2}\right](g \circ \nabla u)(t) \\
& +\left[\frac{M}{2}-\frac{g(0)}{4 \eta}\left(1+\frac{C_{*}^{2}}{\rho+1}\right)\right]\left(g^{\prime} \circ \nabla u\right)(t)-M\left\|u_{t}(t)\right\|_{q+2}^{q+2}+\varepsilon\|u(t)\|_{p+2}^{p+2} \\
& -M \sigma\left(\frac{1}{2} \frac{d}{d t}\|\nabla u(t)\|^{2}\right)^{2}-\left(M-\frac{\varepsilon\|h\|_{\infty}}{4 \eta f_{0}}-\frac{\eta\|h\|_{\infty}}{f_{0}}\right) \int_{\Gamma_{1}} h(x) f(x) y_{t}^{2}(t) d \Gamma \\
& -\varepsilon \int_{\Gamma_{1}} h(x) m(x) y^{2}(t) d \Gamma-\left(M C_{1}-\frac{\varepsilon\left|\mu_{1}\right|}{4 \eta}-\eta\left|\mu_{1}\right|\right) \int_{\Gamma_{1}} u_{t}^{2}(x, t-\tau(t)) d \Gamma \\
& -\frac{M \lambda \xi}{2} \int_{t-\tau(t)}^{t} \int_{\Gamma_{1}} e^{-\lambda(t-s)} u_{t}^{2}(s) d \Gamma d s-\left(M C_{1}-\frac{\varepsilon \mu_{0}}{4 \eta}-\eta \mu_{0}\right) \int_{\Gamma_{1}} u_{t}^{2}(x, t) d \Gamma \\
& -\frac{2(p+2) \sigma}{p} E(0) E^{\prime}(t) \text {. }
\end{aligned}
$$

At this point, we choose $\varepsilon>0$ small enough and we pick $\eta>0$ sufficiently small such that

$$
K_{1}=\frac{1}{\rho+1}\left(g_{0}-\varepsilon\right)>0, \quad K_{2}=g_{0}-\eta\left(\alpha_{2}+\frac{\alpha_{4}}{\rho+1}\right)-\varepsilon\left(\frac{\alpha_{2}}{4 \eta}+1\right)>0,
$$

and then we choose $M$ so large that

$$
\begin{aligned}
K_{3}= & \frac{M g(0)}{2}-\varepsilon(1+\eta)(a-l)-\varepsilon \eta\left(C_{*}^{2}+\mu_{0} \tilde{C}_{*}^{2}+\tilde{C}_{*}^{2}+\left|\mu_{1}\right| \tilde{C}_{*}^{2}\right) \\
& -\eta\left(1+\alpha_{3}\right)-2 \eta(a-l)^{2}>0 \\
K_{4}= & \frac{M}{2}-\frac{g(0)}{4 \eta}\left(1+\frac{C_{*}^{2}}{\rho+1}\right)>0 \\
K_{5}= & M-\frac{\varepsilon\|h\|_{\infty}}{4 \eta f_{0 *}}-\frac{\eta\|h\|_{\infty}}{f_{0 *}}>0 \\
K_{6}= & M C_{1}-\frac{\varepsilon\left|\mu_{1}\right|}{4 \eta}-\eta\left|\mu_{1}\right|>0 \\
K_{7}= & M C_{1}-\frac{\varepsilon \mu_{0}}{4 \eta}-\eta \mu_{0}>0 .
\end{aligned}
$$


Hence, for any $t \geq t_{0}$, we arrive at

$$
\begin{aligned}
L^{\prime}(t) \leq & -K_{1}\left\|u_{t}(t)\right\|_{\rho+2}^{\rho+2}-K_{3}\|\nabla u(t)\|^{2}-\varepsilon\left(a+b\|\nabla u(t)\|^{2}\right)\|\nabla u(t)\|^{2} \\
& -K_{2}\left\|\nabla u_{t}(t)\right\|^{2}+K_{8}(g \circ \nabla u)(t)+K_{4}\left(g^{\prime} \circ \nabla u\right)(t)-M\left\|u_{t}(t)\right\|_{q+2}^{q+2}+\varepsilon\|u(t)\|_{p+2}^{p+2} \\
& -M \sigma\left(\frac{1}{2} \frac{d}{d t}\|\nabla u(t)\|^{2}\right)^{2}-K_{5} \int_{\Gamma_{1}} h(x) f(x) y_{t}^{2}(t) d \Gamma-\varepsilon \int_{\Gamma_{1}} h(x) m(x) y_{t}^{2}(t) d \Gamma \\
& -K_{6} \int_{\Gamma_{1}} u_{t}^{2}(x, t-\tau(t)) d \Gamma-\frac{M \lambda \xi}{2} \int_{t-\tau(t)}^{t} \int_{\Gamma_{1}} e^{-\lambda(t-s)} u_{t}^{2}(s) d \Gamma d s \\
& -K_{7} \int_{\Gamma_{1}} u_{t}^{2}(x, t) d \Gamma-\frac{2(p+2)}{p} E(0) E^{\prime}(t)
\end{aligned}
$$

where

$$
K_{8}=\frac{1}{4 \eta}\left[\varepsilon+(a-l)\left(a+b \frac{2(p+2)}{l p} E(0)\right)^{2}+(a-l)\left(2+3 \tilde{C}_{*}^{2}+8 \eta^{2}+\mu_{0} \tilde{C}_{*}^{2}+\left|\mu_{1}\right| \tilde{C}_{*}^{2}\right]\right.
$$

It follows that

$$
L^{\prime}(t) \leq-K_{9} E(t)+K_{10}(g \circ \nabla u)(t)-\frac{2(p+2)}{p} E(0) E^{\prime}(t)
$$

where $K_{9}$ and $K_{10}$ are some positive constants. Multiplying the above inequality by $\zeta(t)$ and using (2.6) and (3.5), we obtain, for any $t \geq t_{0}$,

$$
\zeta(t) L^{\prime}(t) \leq-K_{9} \zeta(t) E(t)-\left(2 K_{10}+K_{11} \zeta(t)\right) E^{\prime}(t)
$$

where $K_{11}=\frac{2(p+2)}{p} E(0)$.

Now, we define

$$
G(t)=\zeta(t) L(t)+\left(2 K_{10}+K_{11} \zeta(t)\right) E(t)
$$

As $\xi$ is non-increasing positive function, by using Lemma 3.4, the function $G(t)$ is equivalent to $E(t)$. Using the fact that $\xi^{\prime}(t) \leq 0,(3.45)$ implies that

$$
G^{\prime}(t) \leq-K_{9} \zeta(t) E(t) \leq-v \zeta(t) G(t)
$$

where $v$ is a positive constant.

The integration of the inequality between $t_{0}$ and $t$ gives the following estimation for the function $G(t)$ :

$$
G(t) \leq G\left(t_{0}\right) e^{-v \int_{t_{0}}^{t} \zeta(s) d s}, \quad \forall t \geq t_{0}
$$

Again, employing that $G$ is equivalent to $E$, we get

$$
E(t) \leq K e^{-v \int_{t_{0}}^{t} \zeta(s) d s}, \quad \forall t \geq t_{0}
$$

where $K$ is a positive constant. Thus the proof of Theorem 3.2 is completed. 


\section{Competing interests}

The authors declare that they have no competing interests.

\section{Authors' contributions}

The authors declare that the study was realized in collaboration with the same responsibility. All authors read and approved the final manuscript.

\section{Author details}

${ }^{1}$ Department of Mathematics, Pusan National University, Pusan, 46241, South Korea. ${ }^{2}$ Department of Mathematics Education, Seowon University, Cheongju, 28674, South Korea.

\section{Acknowledgements}

The authors would like to express their gratitude to the anonymous referees for a helpful and very careful reading of this paper. The work was supported by the National Research Foundation of Korea (Grant $\sharp$ NRF-2016R1C1B1016288 and Grant $\sharp$ NRF-2013R1A1A2010704)

Received: 13 June 2016 Accepted: 13 September 2016 Published online: 22 September 2016

\section{References}

1. Wu, JQ: Well-posedness for a variable-coefficient wave equation with nonlinear damped acoustic boundary conditions. Nonlinear Anal. 75(18), 6562-6569 (2012)

2. Bucci, F, Lasiecka, I: Exponential decay rates for structural acoustic model with an over damping on the interface and boundary layer dissipation. Appl. Anal. 81(4), 977-999 (2002)

3. Lasiecka, I: Boundary stabilization of a 3-dimensional structural acoustic model. J. Math. Pures Appl. (9) 78(2), 203-232 (1999)

4. Mugnolo, D: Abstract wave equation with acoustic boundary conditions. Math. Nachr. 279(3), 299-318 (2006)

5. Park, JY, Park, SH: Decay rate estimates for wave equation of memory type with acoustic boundary conditions. Nonlinear Anal. TMA 74(3), 993-998 (2011)

6. Beale, JT, Rosencrans, SI: Acoustic boundary conditions. Bull. Am. Math. Soc. 80, 1276-1278 (1974)

7. Beale, JT: Spectral properties of an acoustic boundary condition. Indiana Univ. Math. J. 25(9), 895-917 (1976)

8. Abdallah, C, Dorato, P, Benitez-Read, J, Byrne, R: Delayed positive feedback can stabilize oscillatory system. In: ACC, San Francisco, pp. 3106-3107 (1993)

9. Nicaise, S, Pihnotti, C: Stability and instability results of the wave equation with a delay term in the boundary or internal feedbacks. SIAM J. Control Optim. 45, 1561-1585 (2006)

10. Nicaise, S, Pihnotti, C: Stabilization of the wave equation with boundary or internal distributed delay. Differ. Integral Equ. 21, 935-958 (2008)

11. Xu, CQ, Yung, SP, Li, LK: Stabilization of the wave system with input delay in the boundary control. ESAIM Control Optim. Calc. Var. 12, 770-785 (2006)

12. Balakrishnan, AV, Taylor, LW: Distributed parameter nonlinear damping models for flight structures. In: Proceedings "Daming 89", Flight Dynamics Lab and Air Force Wright Aeronautical Labs, WPAFB (1989)

13. Bass, RW, Zes, D: Spillover nonlinearity and flexible structures. In: The Fourth NASA Workshop on Computational Control of Flexible Aerospace Systems. NASA Conference Publication, vol. 10065, pp. 1-14 (1991)

14. You, Y: Inertial manifolds and stabilization of nonlinear beam equations with Balakrishnan-Taylor damping. Abstr. Appl. Anal. 1, 83-102 (1996)

15. Clark, HR: Elastic membrane equation in bounded and unbounded domains. Electron. J. Qual. Theory Differ. Equ. 2002, 11 (2002)

16. Tatar, N-e, Zarai, A: Exponential stability and blow up for a problem with Balakrishnan-Taylor damping. Demonstr. Math. XLIV(1), 67-90 (2011)

17. Zarai, A, Tatar, N-e: Global existence and polynomial decay for a problem with Balakrishnan-Taylor damping. Arch. Math. 46, 157-176 (2010)

18. $\mathrm{Mu}, \mathrm{C}, \mathrm{Ma}$, J: On a system of nonlinear wave equation with Balakrishnan-Taylor damping. Z. Angew. Math. Phys. 65 91-113 (2014)

19. Lee, MJ, Park, JY, Kang, YH: Asymptotic stability of a problem with Balakrishnan-Taylor damping and a time delay. Comput. Math. Appl. 70, 478-487 (2015)

20. Wu, ST: General decay of solutions for a viscoelastic equation with Balakrishnan-Taylor damping. Taiwan. J. Math. 17(3), 756-784 (2013)

21. Zhang, Z, Huang, J, Liu, Z, Sun, M: Boundary stabilization of a nonlinear viscoelastic equation with interior time-varying delay and nonlinear dissipative boundary feedback. Abstr. Appl. Anal. 2014, 102594 (2014)

22. Boukhatem, Y, Benabderrahmane, B: Existence and decay of solutions for a viscoelastic wave equation with acoustic boundary conditions. Nonlinear Anal. 97, 191-209 (2014)

23. Avalos, G, Lasiecka, I, Rebarber, R: Uniform decay properties of a model in structural acoustics. J. Math. Pures Appl. 79(10), 1057-1072 (2000) 